(C) 2014 IEEE. Personal use of this material is permitted. Permission from IEEE must be obtained for all other uses, in any current or future media, including reprinting/republishing this material for advertising or promotional purposes, creating new collective works, for resale or redistribution to servers or lists, or reuse of any copyrighted component of this work in other works.

This paper appears in: RF and Wireless Technologies for Biomedical and Healthcare Applications (IMWS-Bio), 2014 IEEE MTT-S International Microwave Workshop Series on, Issue Date: 08-10 Dec. 2014.

The definitive version is available online at:

http://dx.doi.org/10.1109/IMWS-BIO.2014.7032388. 


\title{
A Circularly Polarised Antenna for a Wireless Blood-Flow Sensor
}

\author{
Mohamed El Atrash*, Yi Wang ${ }^{\dagger}$ \\ *School of Electrical Communication \& Electronics Systems Engineering \\ October University for Modern Science and Arts University, Wahat Road, 6 October City, Cairo, Egypt \\ Email:mzaky@msa.eun.eg \\ ${ }^{\dagger}$ University of Greenwich, Kent, ME4 4TB, United Kingdom \\ Email: yi.wang@greenwich.ac.uk
}

\begin{abstract}
This paper presents a circularly polarised antenna for determining the speed of blood-flow, using Doppler effects at microwave frequencies. The structure consists of a double-feed patch antenna and a quadrature hybrid coupler in order to achieve circular polarisation and to isolate the reflected signal from the incident. The design was simulated and then fabricated. Both results were compared which showed a good agreement in terms of scattering parameters and radiation patterns.
\end{abstract}

Index Terms - Blood-flow, circular polarisation, human tissue, microstrip patch antenna, quadrature hybrid coupler.

\section{INTRODUCTION}

No one can deny the impact of wireless technologies on our lives and, particularly, their development into medical applications. For instance, wearable wireless sensors have become a promising field of interest, as they can be used to alert users of vital signs such as blood pressure, blood glucose levels and to monitor heart rates [1]. Alongside that, the speed of blood-flow is another indicator of health of the human body, as fluctuations in blood velocity are used as a sign indicating the existence of medical concerns. This is less well known partly because a blood-flow sensor is not readily available. Low blood velocity means that some organs of the body may not get the reasonable amount of needed blood; thereby suffering from de-oxygenation. For example, for stroke patients, the decrease in the speed of the blood flow may lead to the death of cells in the brain due to the fact that they are deprived of the required amount of oxygen. On the other hand, high blood velocity may cause some vessels to burst and hence bleeding. A major factor for causing fluctuations in the speed of the blood-flow is the change of the heart health and blood pressure as reported in [2].

The existing technologies that were demonstrated or applied for measuring blood-flow include Micro-Electro-MechanicalSystems (MEMS) and laser Doppler [3]-[5], magnetic disturbance [6], ultrasound waves [7], and pulse oximetry [8]. The potential advantages of using the RF and antenna approach are the non-invasive nature, safety and low cost. References [9] and [10] reported two such antennas at 29.8 $\mathrm{GHz}$ and $3 \mathrm{GHz}$ respectively. The scope of this research project was to design a double-feed circularly polarised microstrip patch antenna at $2.4 \mathrm{GHz}$. The antenna should separate the transmitted microwave signal into the human body from the reflected signal, which is modulated due to the Doppler effect from the blood flow. Accordingly, the quadrature $\left(90^{\circ}\right)$ hybrid coupler, also referred to as the branch line coupler, was used for duplexing, to achieve the quadrature phase difference between the fed signals and thereby realise the circular polarisation. Since the transmitted and received signals are orthogonally polarised, circular polarisation was considered an optimum choice. The frequency of $2.4 \mathrm{GHz}$ was chosen as a trade-off between the antenna size and the penetration depth. Operating at a higher frequency, for instance $24 \mathrm{GHz}$, would result in a more compact antenna. However, according to [11], the penetration depth would be around only $1 \mathrm{~mm}$, whereas at $2.4 \mathrm{GHz}$, the penetration depth is about $23 \mathrm{~mm}$. This depth is acceptable for detecting the blood-flow in the vessels that are close to the skin surface.

The Doppler effect plays a major role in determining the speed of blood-flow. The Doppler frequency shift depends on the blood velocity, the dielectric constant of the human tissues, the incident frequency, and the angle between the direction of blood flow and the antenna beam. According to [12], the range of speed of blood-flow in arteries is 4.9-19 $\mathrm{cm} / \mathrm{s}$ and that in veins is $1.5-7.1 \mathrm{~cm} / \mathrm{s}$. The human tissues have various dielectric properties. For example, according to [11] at $2.4 \mathrm{GHz}$, the dielectric constant is 38 for dry skin, 43 for wet skin, 5.3 for fat, 53 for muscle and 58 for blood. Considering the penetration depth at $2.4 \mathrm{GHz}$, an effective dielectric constant of 40 is assumed here although more accurate estimation will be made by electromagnetic modelling. If the angle is assumed to be $45^{\circ}$, the Doppler frequency shift is estimated to be $11-43 \mathrm{~Hz}$ from the arteries and $3.4-16.1 \mathrm{~Hz}$ from the veins.

\section{ANTENNA DESIGN}

Fig. 1 shows the circularly polarised antenna, which consists of a patch antenna that is doubly fed at its adjacent sides. The $90^{\circ}$ phase shift was achieved using the quadrature hybrid coupler which consists of four ports, where the two output ports were connected to the patch. The remaining one port is for sending the microwave signal into the human body and the other for receiving the modulated one. The circuit was 
implemented on FR-4 substrate of a dielectric constant of 4.3 and a thickness of $1.6 \mathrm{~mm}$. The patch is $29 \times 29 \mathrm{~mm}$ in size. Quarter wave transformers $(15.07 \mathrm{~mm} \times 0.55 \mathrm{~mm}$ in dimensions) were used between the patch and the coupler to achieve good impedance matching.

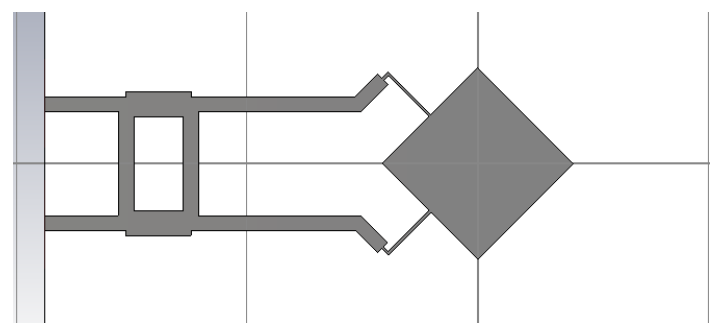

Fig. 1. Circularly polarised microstrip patch antenna design.

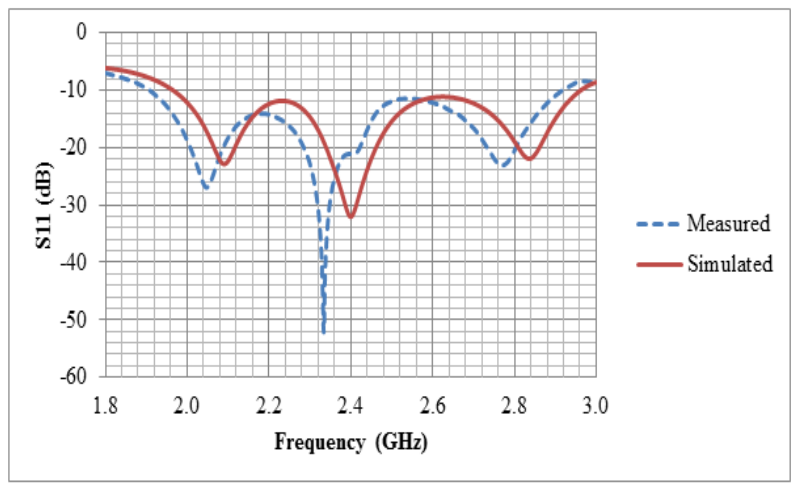

Fig. 2. Simulated versus measured $S_{11}$ response.

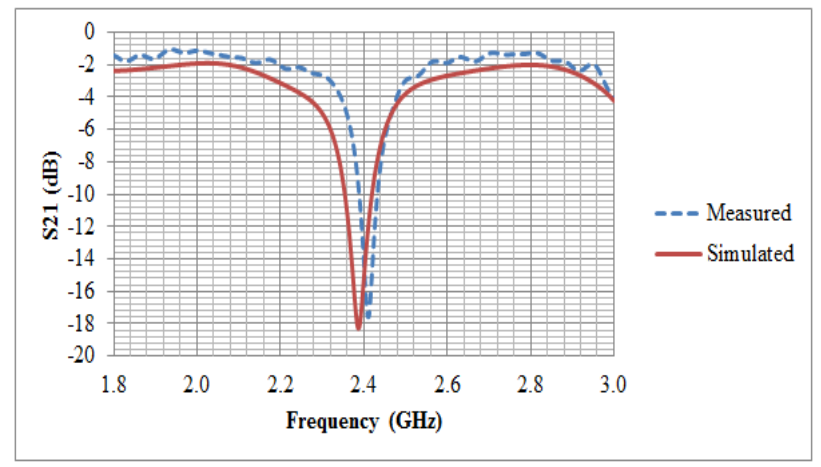

Fig. 3. Simulated versus measured $\mathrm{S}_{21}$ response.

\section{SIMULATION AND MEASUREMENT}

Fig. 2 and Fig. 3 compare the simulated results and the measurements. Good agreement has been achieved. The $S_{11}$ response showed high impedance matching and the $S_{21}$ response showed an acceptable input isolation of $15 \mathrm{~dB}$ at 2.4 GHz. From Fig. 2 and Fig. 3, the insertion loss caused by the FR-4 substrate can be seen. The loss tangent of 0.025 was taken into consideration in the simulation. The simulated directivity of the design was $6.93 \mathrm{dBi}$ at $2.4 \mathrm{GHz}$. However; the gain of the design was only $1.4 \mathrm{~dB}$, which shows that the radiation efficiency is $28 \%$ only. Again, this was due to the fact that the FR-4 substrate material used was very lossy; thereby a lot of the power was dissipated.

Fig. 4 and Fig. 5 show the measured radiation patterns of the fabricated design at different frequencies. Fig. 4 shows the radiation pattern when the linear polarization of the source antenna is parallel to the feed line of the coupler. Fig. 5 shows the radiation pattern when the polarization of the source antenna is vertical to the feed line of the coupler.

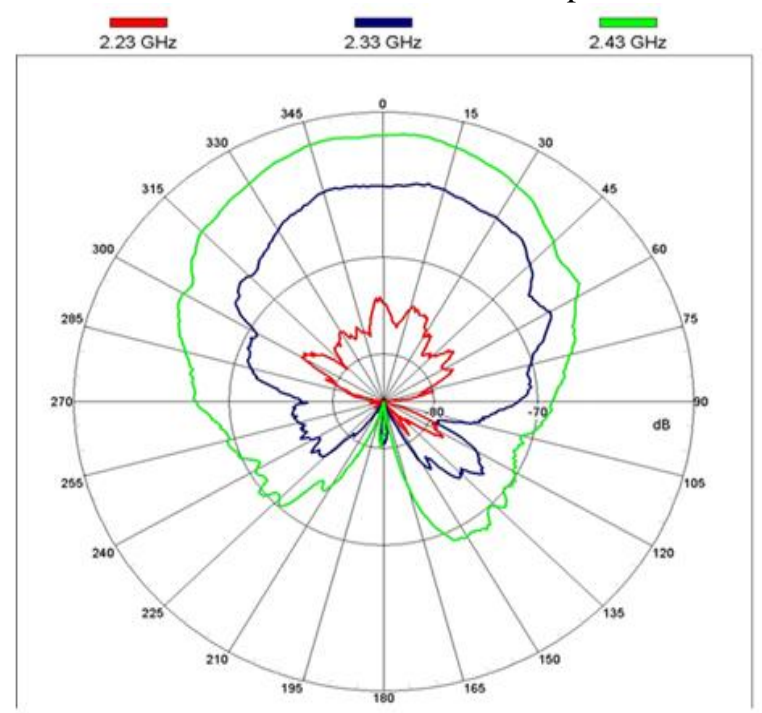

Fig. 4. Measured radiation patterns when the source antenna is vertically polarised.

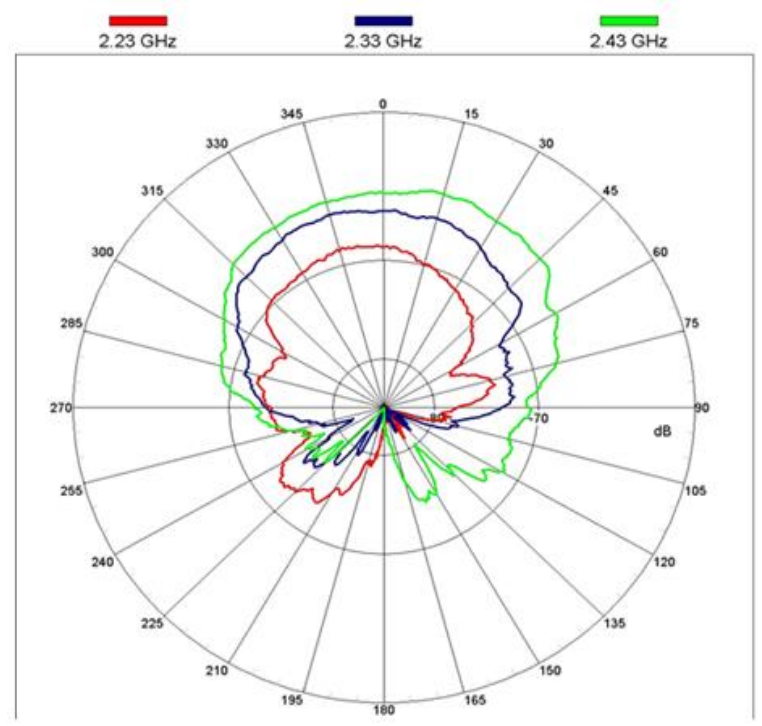

Fig. 5. Measured radiation patterns when the source antenna is horizontalzly polarised. 


\section{CONCLUSION}

A circularly polarised microstrip patch antenna was designed, using CST Microwave Studio software, to operate at $2.4 \mathrm{GHz}$. The challenge was to separate the transmitting signal from the receiving one which was achieved through the use of the hybrid coupler. The microstrip patch antenna was doubly fed at its adjacent edges to realize circular polarisation and connect with the hybrid coupler.

The S-parameters and radiation patterns were measured. Overall, the results showed the ability of the antenna to radiate at the desired resonant frequency, good impedance matching and input isolation. Moreover, the design was fabricated and measured using the network analyser and anechoic chamber, where the measured results were in good agreement with the simulated ones.

The major work that will be implemented in the future is to design an antenna array. This will give a higher gain. More importantly, the array will give the advantage of steering the beam away from the normal without tilting the antenna. This is to maximize the Doppler shift as well as to increase the electromagnetic coupling between the antenna and the human tissue.

\section{REFERENCES}

[1] T. Yilmaz, R. Foster, and Y. Hao, "Detecting vital signs with wearable wireless sensors," Sensors, vol. 10, no. 12, pp. 1083710862, 2010.

[2] M. Maier, and L-G. Lindberg, "Non-invasive blood flow monitoring on the wrist," 2001 Proceedings of the $23^{\text {rd }}$ Annual Engineering in Medicine and Biology Society (EMBS) International Conference of the IEEE, vol. 3, pp. 3034-3035, 2001.

[3] W. Iwasaki, H. Ito, H. Nogami, R. Sawada, Y. Kimura, A. Onoe, S. Takeucchi, M. Furue, and E. Higurashi, "Influence of alcohol consumption on blood flow as detected using a micro integrated laser doppler blood flowmeter," 2010 First International Conference on Sensor Device Technologies and Applications, pp. 236-241, 2010.

[4] H. Nogami, W. Iwasaki, F. Matsuoka, K. Akase, Y. Kimura, A. Onoe, E. Higurashi, S. Takeuchi, M. Kido, M. Furue, and R. Sawada, "Micro integrated laser Doppler blood flow sensor and its application to dehydration prevention," Proceedings of the 2009 IEEE $3^{\text {rd }}$ International Conference on Nano/Molecular Medicine and Engineering, pp. 215-220, 2009.

[5] R. Seto, F. Matsuoka, T. Soh, T. Itoh, H. Okada, T. Masuda, T. Umeda, I. Maeda, K. Tsukamoto, K. Suzuki, Y. Kimura, A. Onoe, E. Higurashi, R. Maeda, W. Iwasaki and R. Sawada, " A micro optical blood flow sensor and its application to detection of avian influenza," Solid-State Sensors, Actuators and Microsystems Conference, TRANSDUCERS 2009, International, pp. 2326-2329, 2009.

[6] C. T, Phua, and G. Lissorgues, "Non-invasive measurement of blood flow using magnetic disturbance method" International Conference on Biomedical and Pharmaceutical Engineering, 2009, ICBPE '09, pp. 1-4, 2009.

[7] E. Awad and H. H. Asada, " The Doppler necklace: a wearable and noninvasive ultrasound sensor for continuous monitoring of blood flow in the common carotid artery," Proceedings of The
First Joint BMES/EMBS Conference Serving Humanity, Advancing Technology, vol. 2, pp. 795, 1999.

[8] S. Rhee, B. Yang, K. Chang, and H. H. Asada, "The ring sensor: a new ambulatory wearable sensor for twenty-four hour patient monitoring," Proceedings of the $20^{\text {th }}$ Annual International Conference of the IEEE Engineering in Medicine and Biology Society, vol. 20, no. 4, pp. 1906-1909, 1998.

[9] F. Shun-yu, L. Zhi-gang, and Z. Shou-zheng, "Ka-band dual circularly polarised transceiver antenna for medical applications," 2012 IEEE MTT-S International, Microwave Workshop Series on Millimeter Wave Wireless Technology and Applications (IMWS), pp 1-4, 2012.

[10] L. Meng-ting, and Z. Shou-zheng, "Compact dual circularly polarized microstrip transceiver antenna for medical applications," 2012 International Conference on Microwave and Millimeter Wave Technology (ICMMT), vol. 1, pp 1-4, 2012.

[11] Italian national research council (2012) IFAC. Available at: http://niremf.ifac.cnr.it/tissprop/ (Accessed: 20 March 2013).

[12] M. Klarhofer, B. Csapo, Cs. Balassy, J. C. Szeles, and E. Moser, "High-resolution blood flow velocity measurements in the human finger," Magnetic Resonance in Medicine, vol. 45, no. 4, pp. 716-719, 2001. 